JGG 2021;69:114-119

doi: $10.36150 / 2499-6564-N 291$

\title{
Age, comorbidities, nursing home stay and outcomes of SARS-CoV-2 infection in a Northern Italian cohort
}

\author{
Francesca Valent \\ Institute of Hygiene and Clinical Epidemiology, University Hospital of Udine, Italy
}

Background \& aims. A large proportion of deaths from SARS-CoV-2 infections worldwide occur among nursing home residents. The aim of this research is to study the association between age and comorbidities and the likelihood of hospitalization and death in patients with SARSCoV-2 infection in an Italian province, taking into account the residential setting.

Methods. Retrospective cohort study based on multiple anonymous administrative health databases. Multinomial logistic regression was used to assess the association of sex, age, comorbidities, nursing home residency, calendar time with likelihood of hospitalization (with no death) and death, adjusting for mutual confounding.

Results. Older subjects had increased likelihood of both outcomes. Congestive heart failure and hypothyroidism significantly increased the likelihood of death. Neurological diseases and hypertension increased the risk of hospitalization. Nursing home residents were over-represented in the cohort. They had reduced likelihood of hospitalization, but mortality was analogous of that of other persons of the same age and clinical conditions.

\author{
Correspondence \\ Francesca Valent \\ SOC Istituto di Igiene ed Epidemiologia Clinica, \\ Azienda Sanitaria Universitaria Friuli Centrale, via \\ Colugna 50, 33100 Udine, Italy \\ Tel. +390542559159 \\ E-mail: francesca.valent@asufc.sanita.fvg.it
}

\section{Conflict of interest}

The Author declares no conflict of interest

How to cite this article: Valent F. Age, comorbidities, nursing home stay and outcomes of SARS-CoV-2 infection in a Northern Italian cohort. Journal of Gerontology and Geriatrics 2021;69:114-119. https://doi. org/10.36150/2499-6564-N291

(C) Copyright by Società Italiana di Gerontologia e Geriatria (SIGG)

\section{(c) (i) $\$$}

\section{OPEN ACCESS}

This is an open access article distributed in accordance with the CC-BY-NC-ND (Creative Commons Attribution-NonCommercial-NoDerivatives 4.0 International) license. The article can be used by giving appropriate credit and mentioning the license, but only for non-commercial purposes and only in the original version. For further information: https://creativecommons.org/licenses/by-nc-nd/4.0/deed.en
Conclusions. Efforts should be done to protect elderly and frail people from SARS-CoV-2, regardless of the residential setting.

Key words: SARS-CoV-2, COVID-19, elderly, comorbidity, nursing home

\section{INTRODUCTION}

Italy, 60 million inhabitants, was strongly hit by the SARS-CoV-2 pandemic, particularly from the end of February to the end of May, totaling more than 240,000 cases of infection and over 30,000 deaths in approximately 4 months ${ }^{1}$. The incidence and consequences of the SARS-CoV-2 outbreak, however, were not homogeneous across the country, with the Northern part of Italy reporting the highest toll.

Older age and chronic comorbidities were associated with poor COVID-19 outcomes worldwide. For example, in China elderly COVID-19 patients with comorbidities were more likely to develop serious or critical illness ${ }^{2}$. In a large Mexican cohort, patients with 3 or more comorbidities had higher risk of hospitalization of ICU admission and case fatality rate ${ }^{3}$. Similarly, in New York City, age and comorbidities of people with COVID-19 predicted hospital admission, in particular, and mortality ${ }^{4}$ and in Atlanta, Georgia, diabetes and elderly age were associated with hospitalization ${ }^{5}$. A special category of elderly and frail people, at high risk of infection and of severe 
outcomes, can be found in nursing homes, where, in many countries, a large proportion of all deaths from COVID-19 occurred ${ }^{6}$. In Sweden, however, approximately two thirds of COVID-19 patients in nursing home are surviving ${ }^{7}$.

In the 530,000-inhabitant province of Udine, in the North-East of Italy, the spread of SARS-CoV-2 was limited if compared with other provinces in the North of the country ${ }^{1}$. Nonetheless, it is estimated that more than 70 people have died from COVID-19 ${ }^{8}$. The population of the province of Udine is older than the country average: mean age is 47.7 years (vs 45.4 in Italy) and the ratio of the population $\geq 65$ to that $\leq 14$ is 2.242 (vs 1.731 in Italy) ${ }^{9}$. In the province, there are over 4000 nursing care beds in more than 30 nursing homes, ranging from less than 10 to approximately 400 beds each. Mean age of persons lining in those facilities is approximately 80 years.

The objective of this study was to assess the association of age and chronic comorbidities with different outcomes of SARS-CoV-2 infection, taking into account to potential confounding effect of residing in a nursing home, in this Italian area.

\section{MATERIALS AND METHODS}

For this retrospective cohort study, we used as the source of information the administrative health databases of Local Health Unit "Friuli Centrale" (ASUFC), geographically corresponding to the 530,000-inhabitant North-Eastern Italian province of Udine. In particular, we analyzed the hospital discharge database, including records of hospitalizations in COVID-19-dedicated wards at the two COVID-19 hospitals in the province; the nursing home database, including dates of admission to and discharge from any nursing home in the province; the list of all healthcare beneficiaries, including sex, date of birth, residence and, when applicable, date of death; and the laboratory database, including results of the real-time reverse-transcriptase polymerase chain reaction (RT-PCR) tests for the detection of SARS-CoV-2 conducted at the University Hospital of Udine. In this Hospital, RT-PCR tests are conducted on upper or respiratory specimens to confirm a diagnosis of COVID-19 in symptomatic patients and on upper respiratory specimens collected though nasopharyngeal or oropharyngeal swabs in asymptomatic persons for screening purposes or during contact tracing activities ${ }^{10}$. In addition, chronic comorbidities were identified through the Johns Hopkins ACG ${ }^{\circledR}$ System (Johns Hopkins ACG System 11.2.1), fed with data from the outpatient care database and the drug prescription databases, in addition to the other above listed databases. Comorbidities considered in this analysis were diabetes, congestive heart failure (CHF), ischemic heart disease $(\mathrm{IHD})$, hypertension, disorders of lipid metabolism, obstructive pulmonary disease (COPD), persistent asthma, osteoporosis, renal failure, rheumatoid arthritis, hypothyroidism; in addition, bipolar disorder, depression, and schizophrenia were collapsed into a broader category of psychiatric disorders; Parkinson disease and seizure disorders were collapsed into neurological disorders. Immunosuppression/transplant and HIV could not be included in the final analysis due to the extremely small number of subjects in these categories. All the above listed databases are anonymous; nonetheless, they can be deterministically linked with each other at the individual level through a stochastic key which is univocal for a given person across all databases.

We built a cohort of all the persons living in the province of Udine who had at least one positive RT-PCR test for SARS-CoV-2 from the beginning of the COVID-19 outbreak in the province (March 1, 2020) to April 15, 2020. All cohort subjects were then followed up to death or April 30, to allow for a minimum follow-up of 15 days, and classified into 4 mutually exclusive groups: a) subjects who died; b) subjects who had a hospitalization requiring an ICU stay; c) subjects who had a hospitalization with no ICU stay; d) subjects with only one or more positive RT-PCR tests for SARS-CoV-2.

For all cohort subjects, we also calculated Charlson's comorbidity index, a weighted index taking into account the number and the seriousness of a person's comorbid diseases and associated with mortality, based on hospitalizations in the 5 years before the positive test for SARS-CoV-2 ${ }^{11}$. The algorithm for calculating Charlson's comorbidity index was also used to identify persons with cancer.

We assessed the association between group and subject's characteristics, including sex, age, comorbidities, and being in a nursing home at the beginning of the COVID-19 local outbreak. At univariate analyses, the statistical significance of the associations were tested through chi-square tests for categorical variables and through one-way ANOVA for normally distributed continuous variables. P-values $<0.05$ were considered statistically significant.

We also built a multinomial logistic regression model to assess the association between group and patient's characteristics, adjusting for the potentially confounding effect of each characteristic on the others. The analysis was also adjusted for time from the local outbreak start and the date of infection diagnosis. Since group B (ICU) was relatively small, in the model hospitalized patients with and without ICU stay were grouped into a single level of the dependent variable, which then had 3 levels. To reduce the number of covariates in the model, the 
stepwise option was used with a significance level of 0.3 required to allow a variable into the model, and a significance level of 0.35 to stay in the model. Odds Ratios (OR) and 95\% Confidence Intervals (95\% Cl) were calculated. An alternative more parsimonious model, including Charlson's comorbidity index instead of all the single comorbidities, was also built to assess the role of age and nursing home residency on hospitalization and death, taking into account the number and seriousness of comorbidities.

Although the dependent variable (group) might resemble an ordinal variable in terms of infection severity, we could not assume that grouping was entirely depending on severity and, consequently, that it was really ordinal. This was also confirmed by some univariate analyses. Thus we decided not to use ordinal logistic regression. All the analyses were conducted using SAS v9.4 (SAS Institute Inc., Cary, NC, USA).

\section{RESULTS}

The cohort included 886 persons. Most cases were identified between March 9 and April 11 (Fig. 1). Of all cases, 70 (7.9\%) died, 16 (1.8\%) had an ICU stay but did not die, 171 (19.3\%) had a hospital stay in a COVID-19 with no need of ICU, and 629 (71.0\%) only had one or more positive RT-PCR test for SARS-CoV-2. 185 subjects (20.9\%) were in nursing homes during the COVID-19 local outbreak. For patients who died, mean time to death was $9.9 \pm 9.6$ days, median 7 days; $75 \%$ of deaths occurred within 12 days from the first positive RT-PCT test.

Table I shows the characteristics of cohort subjects in the 4 groups. Both sex and most comorbidities were

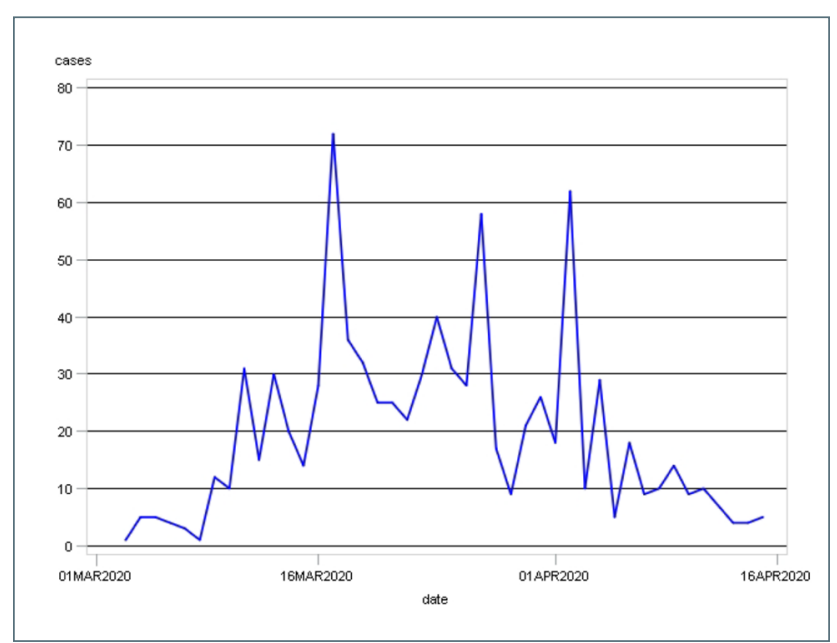

Figure 1. Time of detection of SARS-CoV-2 infected cases in the Italian province of Udine. significantly associated with groups. At univariate analyses, the proportion of hospitalized patients was smaller among females (14.9 with no ICU and $0.8 \%$ with ICU stay) than among males (28.5 and 3.3\%). Among patients with each of the comorbidities of interest except cancer, the proportion of deaths was higher than among those without the specific chronic condition. Charlson's comorbidity index was higher in subjects who died (mean $0.44 \pm 0.86$, median 0) than in those with only positive tests (mean $0.11 \pm 0.58$, median 0 ) and in those hospitalized, either with no need of ICU (mean $0.26 \pm 0.85$, median 0) or with an ICU stay (men and median 0); p-value of Kruskal-Wallis test $<0.0001$ ). In addition, patients with only positive tests were younger (mean age $56.1 \pm 22.1$, median 55 years) than those who were hospitalized, either without ICU (mean age $63.5 \pm 16.0$, median 65 years) or with ICU (mean age $64.6 \pm 10.2$, median 66 years), and $f$ those who died (mean age $84.5 \pm 9.4$, median 85.5 years; $p$-value of ANOVA $<0.0001)$. The proportion of deaths was higher in nursing home residents (25.4\%) than among the other patients (3.3\%).

Table II shows the results of the stepwise multinomial logistic regression model. After adjusting for potentially confounding factors, females positive to SARS-CoV-2 had a reduced likelihood both of hospitalization and of death than males $(\mathrm{OR}=0.502,95 \% \mathrm{Cl}: 0.345-0.731$ and $\mathrm{OR}=0.445,95 \% \mathrm{Cl}: 0.225-0.882$, respectively). Increasing age significantly increased the likelihood of hospitalization and of death: for each additional year, $\mathrm{OR}=1.047,1,95 \% \mathrm{Cl}$ : 034-1.061 and $\mathrm{OR}=1.101$, 95\% Cl: 1.067-1.136, respectively. Although most associations with comorbidities lost statistical significance in the multivariate model, a clearly significant excess in the likelihood of death was associated with $\mathrm{CHF}(\mathrm{OR}=2.606,95 \% \mathrm{Cl}: 1.270-5.345)$. Neurological conditions and hypothyroidism were associated with increased risk of hospitalization $(\mathrm{OR}=2.548,95 \% \mathrm{Cl}$ : 1.079-5.986 and $\mathrm{OR}=2.058,95 \% \mathrm{Cl}: 1.142-3.711$, respectively) but not with death $(\mathrm{OR}=2.173,95 \% \mathrm{Cl}$ : 0.806-5.860 and $\mathrm{OR}=1.104,95 \% \mathrm{Cl}: 0.423-2.841$, respectively). Living in a nursing home strongly reduced the likelihood of being admitted to hospital, whereas no association was found with mortality. Time from start of epidemic to infection detection reduced significantly the likelihood of both hospitalization and death.

When we included Charlson's comorbidity index in the stepwise multinomial logistic regression model instead of the list of distinct comorbidities, the associations of hospitalization and death with sex, age, nursing home residency, and time from start of epidemic were virtually the same as those reported in Table II (data not shown). Adjusting for all those factors, Charlson's comorbidity index was significantly associated with death 
Table I. Demographic characteristics and comorbidities of subjects with SARS-CoV-2 infection living in the Italian province of Udine.

\begin{tabular}{|c|c|c|c|c|c|c|c|c|c|c|}
\hline & & \multicolumn{2}{|c|}{$\begin{array}{c}\text { Positive RT-PCR } \\
\text { test only } \\
(n=629)\end{array}$} & \multicolumn{2}{|c|}{$\begin{array}{c}\text { Hospitalization } \\
\text { (no ICU, no death) } \\
\text { (n= 171) }\end{array}$} & \multicolumn{2}{|c|}{$\begin{array}{c}\text { ICU } \\
\text { (no death) } \\
(n=16)\end{array}$} & \multicolumn{3}{|c|}{$\begin{array}{c}\text { Death } \\
(n=70)\end{array}$} \\
\hline & & $\mathbf{N}$ & $\%$ & $\mathbf{N}$ & $\%$ & $\mathbf{N}$ & $\%$ & $\mathbf{N}$ & $\%$ & P-value \\
\hline \multirow[t]{3}{*}{ Sex } & & & & & & & & & & $<0.0001$ \\
\hline & Female $(n=522)$ & 393 & 75.3 & 78 & 14.9 & 4 & 0.8 & 47 & 9.0 & \\
\hline & Male $(n=364)$ & 236 & 64.8 & 93 & 25.5 & 12 & 3.3 & 23 & 6.3 & \\
\hline \multirow[t]{3}{*}{ Asthma } & & & & & & & & & & 0.1407 \\
\hline & No $(n=814)$ & 585 & 71.9 & 154 & 18.9 & 15 & 1.8 & 60 & 7.4 & \\
\hline & Yes $(n=72)$ & 43 & 61.1 & 17 & 23.6 & 1 & 1.4 & 10 & 13.9 & \\
\hline \multirow[t]{3}{*}{ CHF } & & & & & & & & & & $<0.0001$ \\
\hline & No $(n=805)$ & 592 & 73.5 & 151 & 18.8 & 15 & 1.9 & 47 & 5.8 & \\
\hline & Yes $(n=81)$ & 37 & 45.7 & 20 & 24.7 & 1 & 1.2 & 23 & 28.4 & \\
\hline \multirow[t]{3}{*}{ COPD } & & & & & & & & & & 0.0221 \\
\hline & No $(n=873)$ & 622 & 71.2 & 169 & 19.4 & 16 & 1.8 & 66 & 7.6 & \\
\hline & Yes $(n=13)$ & 7 & 53.8 & 2 & 15.4 & 0 & 0 & 4 & 30.8 & \\
\hline \multirow[t]{3}{*}{ Diabetes } & & & & & & & & & & 0.0102 \\
\hline & No $(n=780)$ & 560 & 71.8 & 152 & 19.5 & 15 & 1.9 & 53 & 6.8 & \\
\hline & Yes $(n=106)$ & 69 & 65.1 & 19 & 17.9 & 1 & 0.9 & 17 & 16.0 & \\
\hline \multirow[t]{3}{*}{ Hypertension } & & & & & & & & & & $<0.0001$ \\
\hline & No $(n=558)$ & 436 & 78.4 & 96 & 17.2 & 6 & 1.1 & 20 & 3.6 & \\
\hline & Yes $(n=328)$ & 193 & 58.8 & 75 & 22.9 & 10 & 3.0 & 50 & 15.2 & \\
\hline \multirow[t]{3}{*}{ Hypothyroidism } & & & & & & & & & & 0.1808 \\
\hline & No $(n=806)$ & 580 & 72.0 & 149 & 18.5 & 15 & 1.9 & 62 & 7.7 & \\
\hline & Yes $(n=80)$ & 49 & 61.2 & 22 & 27.5 & 1 & 1.2 & 8 & 10.0 & \\
\hline \multirow[t]{3}{*}{ IHD } & & & & & & & & & & 0.0030 \\
\hline & No $(\mathrm{n}=839)$ & 604 & 72.0 & 160 & 19.1 & 15 & 1.8 & 60 & 7.1 & \\
\hline & Yes $(n=47)$ & 25 & 53.2 & 11 & 23.4 & 1 & 2.1 & 10 & 21.3 & \\
\hline \multirow{3}{*}{$\begin{array}{l}\text { Disorder of lipid } \\
\text { metabolism }\end{array}$} & & & & & & & & & & 0.0367 \\
\hline & No $(\mathrm{n}=771)$ & 559 & 72.5 & 140 & 18.2 & 15 & 1.9 & 57 & 7.4 & \\
\hline & Yes $(n=115)$ & 70 & 60.9 & 31 & 27.0 & 1 & 0.9 & 13 & 11.3 & \\
\hline \multirow[t]{3}{*}{ Neurological } & & & & & & & & & & 0.0036 \\
\hline & No $(\mathrm{n}=844)$ & 608 & 72.0 & 158 & 18.7 & 16 & 1.9 & 62 & 7,3 & \\
\hline & Yes $(n=42)$ & 21 & 50.0 & 13 & 31.0 & 0 & 0 & 8 & 19.0 & \\
\hline \multirow[t]{3}{*}{ Osteoporosis } & & & & & & & & & & 0.0938 \\
\hline & No $(n=720)$ & 524 & 72.8 & 131 & 18.2 & 13 & 1.8 & 52 & 7.2 & \\
\hline & Yes $(\mathrm{N}=166)$ & 105 & 63.2 & 40 & 24.1 & 3 & 1.8 & 18 & 10.8 & \\
\hline \multirow[t]{3}{*}{ Psychiatric } & & & & & & & & & & 0.0459 \\
\hline & No $(\mathrm{N}=760)$ & 540 & 71.0 & 152 & 20.0 & 16 & 2.0 & 53 & 7.0 & \\
\hline & Yes $(\mathrm{N}=126)$ & 89 & 70.6 & 19 & 15.1 & 1 & 0.8 & 17 & 13.6 & \\
\hline \multirow[t]{3}{*}{ Renal failure } & & & & & & & & & & $<0.0001$ \\
\hline & No $\mathrm{N}=865$ ) & 620 & 71.7 & 167 & 19.3 & 16 & 1.8 & 62 & 7.2 & \\
\hline & Yes $(n=21)$ & 9 & 42.7 & 4 & 19.0 & 0 & 0 & 8 & 38.1 & \\
\hline \multirow{3}{*}{$\begin{array}{l}\text { Rheumatoid } \\
\text { arthritis }\end{array}$} & & & & & & & & & & 0.7861 \\
\hline & No $(n=859)$ & 611 & 71.1 & 165 & 19.2 & 16 & 1.9 & 67 & 7.8 & \\
\hline & Yes $(n=27)$ & 18 & 66.7 & 6 & 22.2 & 0 & 0 & 3 & 11.1 & \\
\hline \multirow[t]{3}{*}{ Cancer } & & & & & & & & & & 0.4807 \\
\hline & No $(n=875)$ & 622 & 71.1 & 167 & 19.1 & 16 & 1.8 & 70 & 8.0 & \\
\hline & Yes $(n=11)$ & 7 & 63.6 & 4 & 36.4 & 0 & 0 & 0 & 0 & \\
\hline Nursing home & & & & & & & & & & $<0.0001$ \\
\hline & No $(n=701)$ & 500 & 71.3 & 162 & 23.1 & 16 & 2.3 & 23 & 3.3 & \\
\hline & Yes $(n=185)$ & 129 & 69.7 & 9 & 4.9 & 0 & 0 & 47 & 25.4 & \\
\hline
\end{tabular}


Table II. Results of stepwise multinomial logistic regression model for demographic characteristics and comorbidities of subjects with SARS-CoV-2 infection living in the Italian province of Udine.

\begin{tabular}{|l|c|c|c|c|}
\hline & \multicolumn{2}{|c|}{ Hospitalization vs positive test } & \multicolumn{2}{c|}{ Death vs positive test } \\
\hline Characteristic & $\mathbf{O R}$ & $\mathbf{9 5 \%} \mathbf{~ C l}$ & $\mathbf{0 R}$ & $\mathbf{9 5 \%} \mathbf{~ C l}$ \\
\hline Female vs male & 0.502 & $0.345-0.731$ & 0.445 & $0.225-0.882$ \\
\hline Age (continuous, in years) & 1.047 & $1.034-1.061$ & 1.101 & $1.067-1.136$ \\
\hline COPD & 0.973 & $0.145-6.548$ & 4.113 & $0.829-20.404$ \\
\hline CHF & 1.303 & $0.648-2.618$ & 2.606 & $1.270-5.345$ \\
\hline Hypertension & 0.949 & $0.619-1.453$ & 1.756 & $0.909-3.393$ \\
\hline Hypothyroidism & 2.058 & $1.142-3.711$ & 1.104 & $0.429-2.841$ \\
\hline Neurological comorbidity & 2.542 & $1.079-5.986$ & 2.173 & $0.806-5.860$ \\
\hline Nursing home & 0.057 & $0.026-0.124$ & 1.069 & $0.493-2.318$ \\
\hline Days from start of outbreak to diagnosis & 0.977 & $0.958-0.996$ & 0.944 & $0.914-0.975$ \\
\hline
\end{tabular}

$(\mathrm{OR}=1.392,95 \% \mathrm{Cl}: 1.043-1.858)$ but not with hospitalization (OR $=1.173,95 \% \mathrm{Cl}: 0.912-1.510)$.

\section{DISCUSSION}

This study assessed the association between demographic and clinical characteristics of persons with a SARS-CoV-2 infection and the likelihood of being admitted to the hospital or dying in the 530,000-inhabitant Italian province of Udine. In the cohort of almost 900 persons diagnosed with SARS-CoV-2, those living in a nursing home were over-represented (20\%), indicating that prevalence of infection was particularly high in this setting. Thus, assessments of the association between chronic comorbidities and outcomes cannot overlook a person's residential situation.

Taking nursing home into account, in our area, age and sex of patients infected by SARS-CoV-2 were significantly associated with increased risk of both hospitalization and death. Among all the comorbidities of interest, CHF and hypothyroidism were significantly associated with increased likelihood of death; patients with neurological conditions, hypertension, and COPD also had increased risk of death, although the association did not reach statistical significance. Neurological patients and those with hypothyroidism had increased risk of hospitalization with alive discharge. Increasing Charlson's comorbidity index also increased the risk of death from COVID-19.

Several risk factors were proposed to explain the high risk of death from COVID-19 among elderly patients, including comorbidities ${ }^{12}$. In fact, not only are the elderly exposed to the effects of immunosenescence, of the continuous production of inflammatory mediators and cytokines, and of aberrant ciliary function and ciliary ultrastructural anomalies, which contribute to the increased mortality from COVID-19, but they may have multiple comorbidities and polypharmacotherapy which can both contribute directly to poor outcomes and complicate COVID-19 therapeutic management ${ }^{12}$. Elderly subjects in nursing homes, where living conditions make viral transmission easy, cause great concern ${ }^{12}$. In this study, nursing home residents had decreased likelihood of being hospitalized (and being discharged alive), meaning that there was a tendency to take care of infected patients directly in the nursing home, instead of transferring them to hospitals. Otherwise, nursing home residents who were hospitalized might have had an unfavorable outcome. This management of nursing home residents did not necessarily mean that this patients received less or poorer care than the others. In fact, the likelihood of dying for nursing home residents was similar to that of the other COVID-19 patients of the same age and with the same comorbidities. In the province of Udine, three quarters of COVID-19 patients in nursing homes were surviving as after the first two months of epidemic, a proportion even higher than in Sweden ${ }^{7}$. However, since case fatality was high in general, nursing homes accounted for two thirds of all COVID-19 deaths in whole province because the high prevalence of infection in such peculiar setting. Thus, it is of the utmost importance that all possible measures are taken to prevent the virus circulation, staff training and access to personal protective equipment, and staff and resident periodic testing ${ }^{6}$.

The multinomial model showed that the likelihood of hospitalization and, in particular, of death decreased as time from outbreak start and diagnosis of infection increased, indicating that the level of care provided to patients has increased. Improvement of care over time was also reported in New York City ${ }^{4}$.

Results of this analysis should be interpreted in the light of some limitations. First, cohort size was relatively small, making association estimates quite imprecise. In addition, only all-cause mortality was available at the 
time the study was conducted, thus it was not possible to distinguish COVID-19-related deaths from other deaths. Nonetheless, since most all deaths occurred in the days or weeks immediately following a COVID-19 diagnosis, it likely that SARS-CoV-2 had some role in all those deaths.

This analysis had also a great advantage: it was entirely conducted using anonymous databases which are timely available, cover the entire population, and can be linked through stochastic keys.

In conclusion, in this Italian context, older persons and those affected by some chronic conditions suffered worse consequences in case of SARS-CoV-2 infection. We must protect frail patients in case of further COVID-19 waves, regardless of the residential setting.

\section{ETHICAL APPROVAL}

Not required. The analyses were based on anonymous administrative data, therefore patient consent and Ethical Committee approval were not required in Italy.

\section{Ethical STATEMENT}

All procedures contributing to this work comply with the Declaration of Helsinki. The analyses were based on anonymous administrative data, therefore patient consent and Ethical Committee approval were not required in Italy.

\section{FUNDING}

This research received no specific grant from any funding agency in the public, commercial, or not-for-profit sectors.

\section{References}

1 Dipartimento della Protezione Civile. Aggiornamento casi COVID-19 (http://opendatadpc.maps.arcgis. com/apps/opsdashboard/index.html\#/b0c68bce2cce478eaac82fe38d4138b1, accessed 10 July 2020).

2 Yang A, Qiu Q, Kong X, et al. Clinical and epidemiological characteristics of COVID-19 patients in Chongqing China. Front Public Health 2020;8:244. https://doi.org/10.3389/ fpubh.2020.00244
3 Kammar-García A, Vidal-Mayo JJ, Vera-Zertuche JM, et al. Impact of comorbidities In Mexican SARS-Cov-2-positive patients: a retrospective analysis in a national cohort. Rev Invest Clin 2020;72:151-8. https://doi.org/10.24875/ RIC.20000207

4 Petrilli CM, Jones SA, Yang J, et al. Factors associated with hospital admission and critical illness among 5279 people with coronavirus disease 2019 in New York City: prospective cohort study. BMJ 2020;369:m1966. https:// doi.org/10.1136/bmj.m1966

5 Killerby ME, Link-Gelles R, Haight SC, et al. Characteristics associated with hospitalization among patients with COVID-19 - Metropolitan Atlanta, Georgia, March-April 2020. MMWR Morb Mortal Wkly Rep 2020;69:790-4. https://doi. org/10.15585/mmwr.mm6925e1

6 ECDC Public Health Emergency Team, Danis K, Fonteneau L, et al. High impact of COVID-19 in long-term care facilities, suggestion for monitoring in the EU/EEA, May 2020. Euro Surveill 2020;25:2000956. https://doi. org/10.2807/1560-7917.ES.2020.25.22.2000956

7 Amér S, Molnar C, Tuutma M, et al. Närmare två tredjedelar av covid-sjuka på SÄBO överlever [Almost two-thirds of the elderly with covid-19 surviving in nursing homes]. Lakartidningen 2020;117:20104.

8 Regione autonoma Friuli Venezia Giulia. Protezione Civile della Regione (https://covid19map.protezionecivile.fvg.it, accessed 10 July 2020).

9 Istat. Indicatori demografici (http://dati.istat.it/Index. aspx?DataSetCode=DCIS_INDDEMOG1, accessed 10 July 2020).

10 World Health Organization. Laboratory testing for coronavirus disease (COVID-19) in suspected human cases (https://www.who.int/publications-detail/laboratory-testing-for-2019-novel-coronavirus-in-suspected-humancases-20200117, accessed March 25, 2020).

11 Charlson ME, Pompei P, Ales KL, MacKenzie CR. A new method of classifying prognostic comorbidity in longitudinal studies: development and validation. J Chronic Dis 1987;40:373-83. https://doi.org/10.1016/0021 9681(87)90171-8

12 Perrotta F, Corbi G, Mazzeo G, et al. COVID-19 and the elderly: insights into pathogenesis and clinical decisionmaking. Aging Clin Exp Res 2020;32:1599-608. https:// doi.org/10.1007/s40520-020-01631-y 